\title{
MIMO Radar Ambiguity Optimization using Phase Coded Pulse Waveforms
}

\author{
G.V.K.Sharma \\ Department of ECE, GITAM Institute of Technology, \\ GITAM University, Visakhapatnam
}

\author{
K. Raja Rajeswari \\ Department of ECE, College of Engineering, \\ Andhra University, Visakhapatnam
}

\begin{abstract}
Multiple input multiple output radars transmit partially coherent or noncoherent waveforms for improving spatial resolution or spatial diversity. While waveform design for SIMO radars concentrate on improving the delay and Doppler resolution characteristics, waveform design for MIMO radars involve optimization of delay, Doppler and spatial resolution characteristics. The resolution properties of MIMO radar transmit waveforms is studied using the MIMO radar ambiguity function. MIMO radar ambiguity function of frequency hopping waveforms is recently derived. Considering the many advantages of phase coded pulse waveforms in radar applications, this paper derives the MIMO radar ambiguity function of phased coded pulse waveforms. Further, a numerical optimization algorithm based on simulated annealing is proposed for designing parameters of the phase coded pulse waveforms that minimize the peak of the ambiguity function at all mismatched values of delay, Doppler and angular dimensions.
\end{abstract}

\section{General Terms}

Signal Processing, Radar Signal Processing, Simulated Annealing, Evolutionary Algorithms.

\section{Keywords}

MIMO radar; ambiguity function; pulse coded waveforms; spatial resolution

\section{INTRODUCTION}

Radar systems transmit electromagnetic energy into free space and use the reflected energy from the objects to detect the range, velocity of the desired objects. Modern radar systems employ multiple transmit antenna elements on transmit and multiple receive antenna elements on receive. Multiple transmit antenna elements allow directive radiation of transmit power and multiple receive antenna elements allow directive reception of reflected echoes. These systems further allow angular parameters of the target to be determined. Conventional phased array radars transmit fully coherent waveforms (possibly scaled by a complex constant) from their $\mathrm{M}$ different transmit antenna elements forming a strong transmit beam in the desired direction. Beamforming is performed only by the receive array (containing $\mathrm{N}$ antenna elements) to estimate the angular parameters of the target. Thus the transmit degrees of freedom are limited to one and receive degrees of freedom are $\mathrm{N}$. However multiple input multiple output (MIMO) radars transmit diverse waveforms from their different transmit antenna elements and use joint processing of the received signals from the different receive array elements. While phased array radars employ only spatial diversity, MIMO radars employ both spatial and waveform diversity to improve many aspects of system performance. MIMO radars can employ widely spaced antennas [1] or collocated antennas [2]. While the former configuration offers improved spatial diversity to improve target detection capabilities the latter configuration improves the spatial resolution, parameter identifiability and interference rejection capability. This paper deals with collocated MIMO radar configuration only.

Several waveforms [6] have been designed for single input single output (SISO) radars with wide range of delay and Doppler resolution characteristics. Design of radar waveforms with an information theoretic perspective is proposed in [3]. The choice of waveforms for MIMO radars affect the range, Doppler and spatial resolution characteristics. While waveforms for SISO radar are designed for desired delay and Doppler resolution characteristics, waveforms chosen for MIMO radars should have desirable ambiguity properties in the delay, Doppler and spatial dimensions. The resolution properties of the transmit waveforms is studied using the concept of ambiguity function [6]. The ambiguity function of a transmit waveform represents the output of the matched filter in the presence of delay and Doppler mismatch. For high delay and Doppler resolution, the ambiguity function should resemble a "thumbtack" function around zero delay and Doppler mismatch. The concept of ambiguity function is extended to the MIMO system case by Fuhrmann [4]. Chen and Vaidyanathan [5] have derived the properties of MIMO ambiguity function and further extended the MIMO ambiguity function for frequency hopping waveforms.

Phase coded pulse waveforms are good candidates for radar waveforms since they offer the high time-bandwidth product, have constant modulus and can be easily generated and processed. Several phase coded waveforms have been proposed previously [7]-[10] with good autocorrelation and crosscorrelation properties. These waveforms are designed for good delay and Doppler resolution characteristics but spatial resolution characteristics are not considered. However designing the phase coded waveforms by optimizing the MIMO ambiguity function improves the delay, Doppler and also spatial resolution characteristics.

In this paper, ambiguity function of MIMO radar employing phase coded pulse waveforms is derived. The MIMO radar ambiguity function is expressed in terms of the parameters of the phase coded pulse waveforms. These parameters are further optimized using a simulated annealing algorithm that provides good resolution in the delay, Doppler and spatial dimensions. Section 2 describes MIMO radar signal model and structure of phase coded waveforms. Section 3 derives the MIMO radar ambiguity function employing phase coded pulse waveforms. Section 4 presents the design of parameters of phase coded pulse waveforms using the simulated annealing algorithm. Section 5 presents the numerical results and Section 6 concludes the paper.

\section{MIMO RADAR SYSTEM MODEL}

Consider a monostatic MIMO radar that contains $M$ transmitters and $N$ receivers with their antennas configured 
as uniform linear arrays (ULAs). A point target is assumed and also that the target, transmitters and receivers lie in the same 2-D plane (see Fig. 1). Let $d_{T}$ and $d_{R}$ represent the spacing between consecutive transmitters and receivers respectively, and let $\gamma=d_{T} / d_{R}$. Define the spatial frequency of the target as $f=d_{R} \sin (\theta) / \lambda$, where $\theta$ is the target angle with respect to the broadside direction and $\lambda$ is the carrier wavelength of the transmitted waveforms.

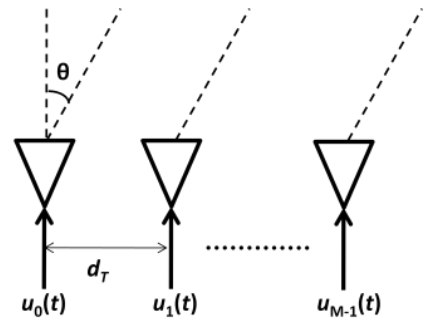

a) Transmitter Model

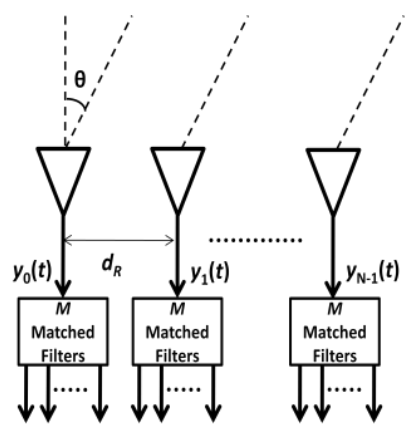

b) Receiver Model

Figure 1: Transmitter and Receiver Models

Let $\tau$ and $v$ be the target delay and Doppler frequency respectively. Let $\left\{u_{m}(t)\right\}, m \in\{0,1, \ldots, M-1\}$ represent the $M$ transmitter waveforms. Then the waveform received at the $n^{\text {th }}$ receiver antenna can be expressed as [5]

$$
y_{n}^{\tau, v, f}(t) \approx \sum_{m=0}^{M-1} u_{m}(t-\tau) e^{j 2 \pi v t} e^{j 2 \pi f(\gamma m+n)}
$$

for $n=0,1, \ldots, N-1$.

\subsection{Phase Coded Pulse Waveforms}

The phase coded pulse waveform emitted by the $m^{\text {th }}$ transmitter can be represented as

$$
u_{m}(t)=\sum_{l=0}^{L-1} \phi_{m}\left(t-T_{l}\right)
$$

where

$$
\begin{gathered}
\phi_{m}(t)=\frac{1}{\sqrt{T_{p}}} \sum_{q=0}^{Q-1} c_{m, q} \mathrm{~s}\left(\frac{t-q \Delta t}{\Delta t}\right) \\
\mathrm{s}(t)= \begin{cases}1 & \text { if } 0<t<1 \\
0 & \text { otherwise }\end{cases}
\end{gathered}
$$

Here $L$ represents the number of pulses emitted by each transmitter. Here, $c_{m, q}$ is the $(m, q)^{\text {th }}$ element of the code matrix $[\mathbf{C}]_{M \times Q}$ and it can assume a value from the set $\left\{e^{j \frac{2 \pi}{K} 0}, e^{j \frac{2 \pi}{K} 1}, \ldots, e^{j \frac{2 \pi}{K}(K-1)}\right\} . T_{p}=Q \Delta t$ is the duration of each pulse and $\Delta t$ is the duration of each subpulse. $K$ is the phase number and represents the number of phases allowed by each polyphase waveform. Each row of the code matrix C represents the phase code associated with each transmitted waveform. Each column of the code matrix corresponds to the phase code transmitted by each of the $M$ transmitters during the $q^{\text {th }}$ subpulse. As shown in Fig. 2, each transmitter waveform $u_{m}(t)$ consists of a stream of $L$ identical pulses $\phi_{m}(t)$. Each pulse in turn contains $Q$ phase coded subpulses each having width $\Delta t$. For each of the transmitter waveforms $u_{m}(t)$ to be orthogonal (at zero Doppler and zero delay mismatch) i.e.,

we require

$$
\int_{-\infty}^{\infty} u_{m}(t) u_{m^{\prime}}^{*}(t) d t=0, \quad \forall m \neq m^{\prime}
$$

$$
\mathbf{C C}^{T}=\mathrm{I}_{M \times M}
$$

Orthogonal waveforms result in uniform illumination in all directions. For fixed $\Delta t$, these waveforms can be completely described by the code matrix $\mathbf{C}=\left[c_{m, q}\right]_{M \times Q}$ and the pulse spacings $\left(T_{0}, T_{1}, \ldots T_{L-1}\right)$. The pulse spacings affect the Doppler resolution of the waveforms.

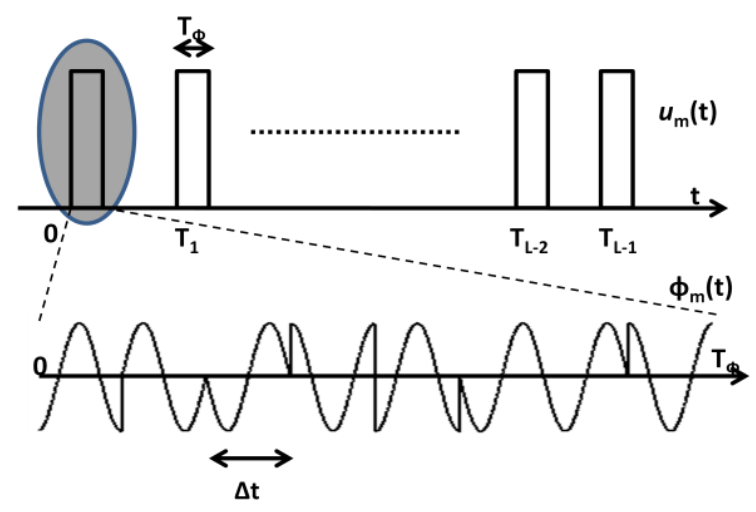

Figure 2: Structure of Phase-Coded Pulse waveforms

\section{MIMO RADAR AMBIGUITY FUNCTION}

The resolution of a radar system is determined by the response to a point target in the matched filter output. This response can be characterized by a function called the ambiguity function. The ambiguity function for SIMO radar is given as

$$
\chi(\tau, v)=\int_{-\infty}^{\infty} u(t) u^{*}(t-\tau) e^{-j 2 \pi v t} d t
$$

Here $\tau$ and $v$ represent the delay and Doppler mismatch at the receiver respectively. The ideal ambiguity function should have a "thumbtack" shape around the zero-mismatch region i.e $(\tau, v)=(0,0)$ so that good delay and Doppler resolution are achieved. The ambiguity function for MIMO case is derived in [4]. Consider the expression for the received signal in MIMO radar given in (2). Let $\left(\tau_{1}, v_{1}, f_{1}\right)$ represent the true parameters of the target and $\left(\tau_{2}, v_{2}, f_{2}\right)$ be the assumed parameters at the receiver. The summed matched filter output is given as

$$
\begin{aligned}
\sum_{n=0}^{N-1} \int_{-\infty}^{\infty} y_{n}^{\tau_{1}, v_{1}, f_{1}}(t) & \left(y_{n}^{\tau_{2}, v_{2}, f_{2}}\right)^{*}(t) d t \\
& =\left(\sum_{n=0}^{N-1} e^{j 2 \pi\left(f_{1}-f_{2}\right) n}\right)
\end{aligned}
$$




$$
\begin{aligned}
\times\left(\sum_{m=0}^{M-1} \sum_{m^{\prime}=0}^{M-1} \int_{-\infty}^{\infty} u_{m}\left(t-\tau_{1}\right) u_{m}^{*}\left(t-\tau_{2}\right)\right. \\
\left.\times e^{j 2 \pi\left(v_{1}-v_{2}\right) t} e^{j 2 \pi\left(f_{1} m-f_{2} m^{\prime}\right) \gamma}\right)
\end{aligned}
$$

$$
\Gamma_{1}=e^{j 2 \pi v q \Delta t} \chi_{\text {rect }}\left(\tau+\left(q-q^{\prime}\right) \Delta \mathrm{t}, v\right)
$$

where $\chi_{\text {rect }}(\tau, v)$ given by

$$
\begin{aligned}
& \chi_{r e c t}(\tau, v)=\int_{0}^{\Delta t} s(t) s(t+\tau) e^{j 2 \pi v t} d t \\
& =\left\{\begin{array}{cc}
\left(\frac{\Delta t-|\tau|}{\Delta t}\right) \operatorname{sinc}(v(\Delta t-|\tau|)) e^{j \pi v(\tau+\Delta t)} & \text { for }|\tau|<L \\
0 & \text { otherwis }
\end{array}\right.
\end{aligned}
$$

denotes the triangular ambiguity function of a rectangular pulse $s(t)$ and represents the output of a matched filter for a single pulse. By substituting (18) into (16), the cross ambiguity function can be written as

$$
\begin{gathered}
\chi_{m, m^{\prime}}^{\phi}(\tau, v)=\frac{1}{T_{p}} \sum_{q=0}^{Q-1} \sum_{q^{\prime}=0}^{Q-1} c_{m, q} c_{m^{\prime} q^{\prime}}^{*} \times e^{j 2 \pi v q \Delta t} \chi_{\text {rect }}(\tau \\
\left.+\left(q-q^{\prime}\right) \Delta \mathrm{t}, v\right)
\end{gathered}
$$

Utilizing the relation $p=q-q^{\prime}$ and collecting the terms centered at the same shift $\tau=p \Delta t$ the double sum in (21) can be written as

$$
\begin{aligned}
\sum_{q=0}^{Q-1} \sum_{q^{\prime}=0}^{Q-1}=\sum_{p=0}^{Q-1} \sum_{q^{\prime}=0}^{Q-1} & \left.\right|_{q=q^{\prime}+p} \\
& +\left.\sum_{p=-(Q-1)} \sum_{q^{\prime}=0}^{Q-1-|p|}\right|_{q^{\prime}=q-p}
\end{aligned}
$$

The cross-ambiguity function $\chi_{m, m^{\prime}}^{\phi}(\tau, v)$ between the phase coded pulses $\phi_{m}(t)$ and $\phi_{m}(t)$ can then be written with (22) as a series of shifted ambiguity functions $\chi_{\text {rect }}(\tau, v)$ of the rectangular pulse as

$$
\begin{aligned}
& \frac{1}{T_{p}} \sum_{p=0}^{Q-1} \chi_{r e c t}(\tau+p \Delta t, v) e^{j 2 \pi v p \Delta t} . S_{1} \\
& +\frac{1}{T_{p}} \sum_{p=0}^{Q-1} \chi_{r e c t}(\tau+p \Delta t, v) . S_{2} \\
& S_{1}=\sum_{q^{\prime}=0}^{Q-1-p} c_{m,\left(q^{\prime}+p\right)} c_{m^{\prime}, q^{\prime}}^{*} e^{j 2 \pi v q^{\prime} \Delta t} \\
& S_{2}=\sum_{q=0}^{Q-1-|p|} c_{m, q} c_{m,(q-p)}^{*} e^{j 2 \pi v q \Delta t}
\end{aligned}
$$

Equations (9), (14), (23) and (24) constitute the complete MIMO ambiguity function for phase coded pulse waveforms. The objective of phase coded pulse waveform design for MIMO radars is to design the code matrix Csuch that the cost function

$$
g_{p}(\mathbf{C}) \triangleq \int_{-\infty}^{\infty} \int_{-\infty}^{\infty} \int_{0}^{1} \int_{0}^{1}\left|\chi_{m, m^{\prime}}\left(\tau, v, f_{1}, f_{2}\right)\right|^{p} d \tau d v d f_{1} d f_{2}
$$

is minimized. To obtain good resolutions, the peaks in $\chi_{m, m^{\prime}}\left(\tau, v, f_{1}, f_{2}\right)$ need to be minimized which are not along the line $\left(0,0, f_{1}, f_{1}\right)$.Typically $p$ is chosen to be 3 so that sidelobe peak values of the MIMO radar ambiguity function at $\tau \neq 0, v \neq 0, f_{1} \neq f_{2}$ are minimized. This forces the energy of the function $\chi_{m, m^{\prime}}\left(\tau, v, f_{1}, f_{2}\right)$ to the evenly spread in the delay, Doppler and angular dimensions.

where

$$
\Gamma_{1}=\int_{-\infty}^{\infty} s\left(\frac{t-q \Delta t}{\Delta t}\right) s\left(\frac{t+\tau-q^{\prime} \Delta t}{\Delta t}\right) e^{j 2 \pi v t} d t
$$

In order to solve (17), the change of variables is made as $t_{1}=t-q \Delta t$, and then integrate over the range $(-\infty, \infty)$. 


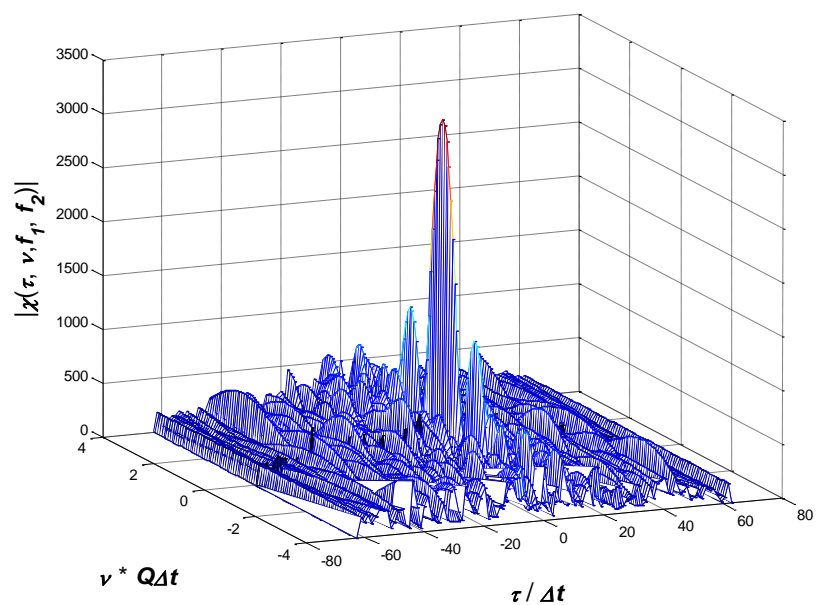

Figure 3: MIMO radar ambiguity function of Phase Coded waveforms generated using sequences designed in Table-1 with $M=5, Q=63, K=4, f_{1}=0, f_{2}=0$

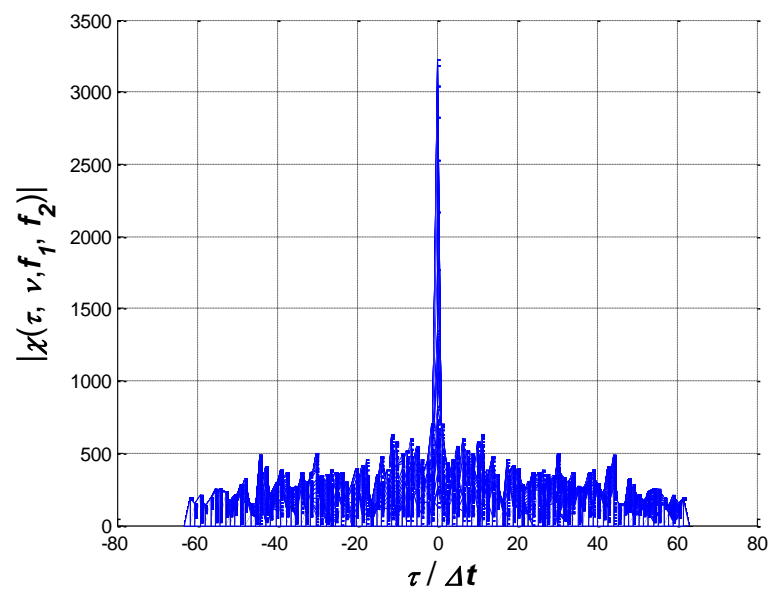

Figure 4: Delay cut of the MIMO radar ambiguity function of Phase Coded waveforms generated using sequences designed in Table-1 with $M=5, Q=63, K=$ $4, f_{1}=0, f_{2}=0$

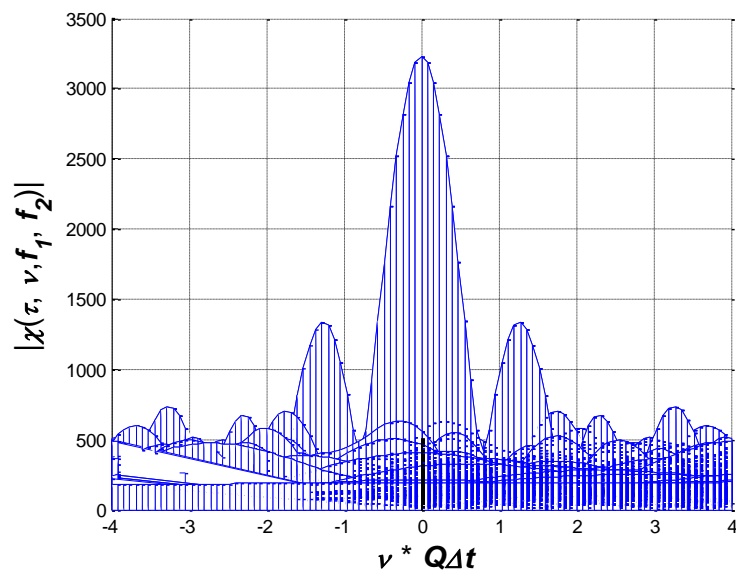

Figure 5: Doppler cut of the MIMO radar ambiguity function of Phase Coded waveforms generated using sequences designed in Table-1 with $M=5, Q=63, K=4$, $f_{1}=0, f_{2}=0$

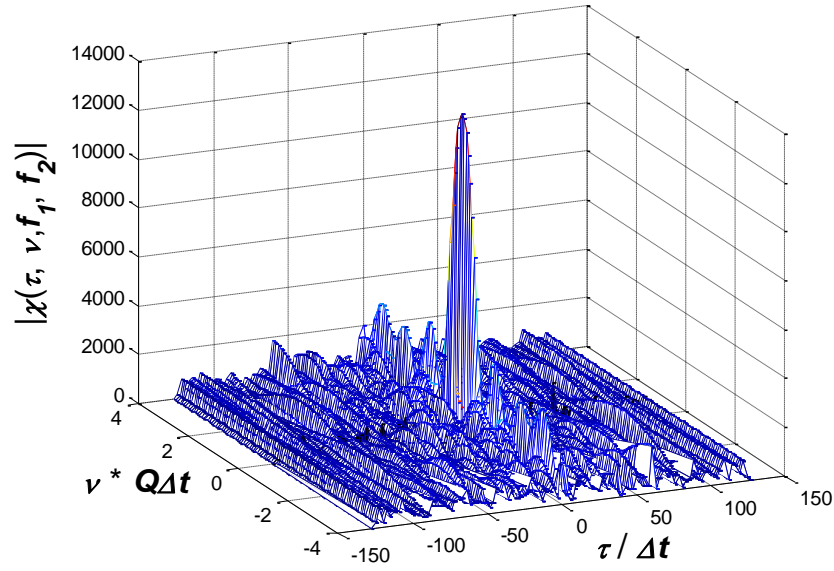

Figure 6: MIMO radar ambiguity function of Phase Coded waveforms generated using sequences designed in Table-2 with $M=16, Q=127, K=4, f_{1}=0, f_{2}=0$

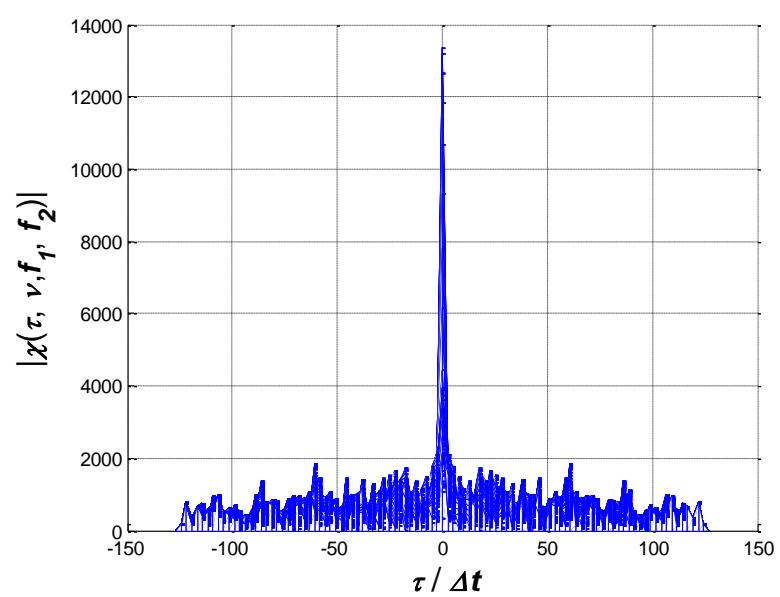

Figure 7: Delay cut of the MIMO radar ambiguity function of Phase Coded waveforms generated using sequences designed in Table-2 with $M=16, Q=$ 127, $K=4, f_{1}=0, f_{2}=0$

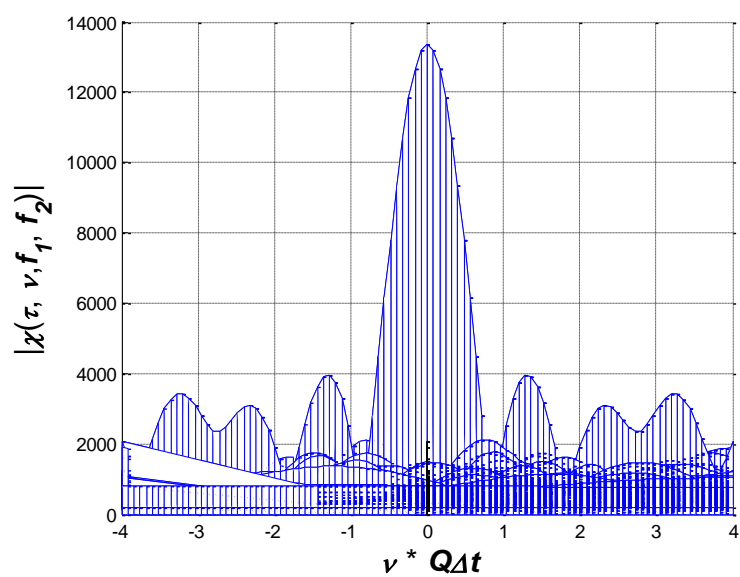

Figure 8: Doppler cut of the MIMO radar ambiguity function of Phase Coded waveforms generated using sequences designed in Table- 2 with $M=16, Q=127, K=$ 4, $f_{1}=0, f_{2}=0$ 

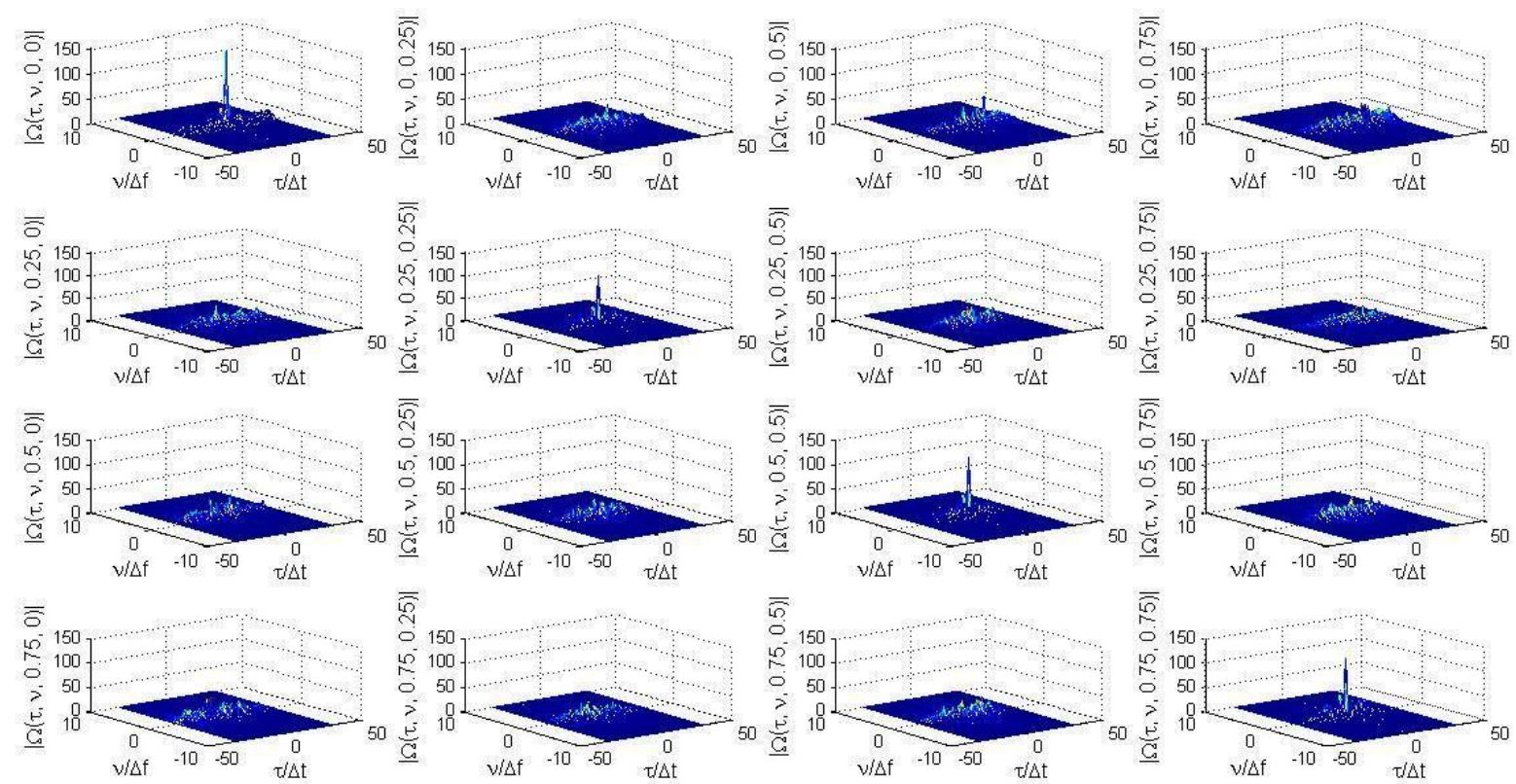

Figure 9: Plots of MIMO radar ambiguity function at various values of $f_{1}$ and $f_{2}$, with $M=5, Q=63$ and $K=4$

\section{CONCLUSIONS}

Considering the many advantages that phase coded waveforms offer over frequency hopping waveforms in terms of better resolution properties, this paper derived the MIMO ambiguity function of phase coded pulse waveforms. Further, a numerical optimization algorithm based on simulated annealing is proposed for designing parameters of the phase coded pulse waveforms that minimize the peak of the ambiguity function at all mismatched values of delay, Doppler and angular dimensions. Phase coded waveforms with good delay, Doppler and spatial resolution properties have been designed using the proposed algorithm. It is seen that that phase coded waveforms offer improved resolution performance over frequency hopping waveforms (in delay, Doppler and spatial dimensions) even when large number of transmit antennas (and hence large number of waveforms) are employed. The choice of phase coded or frequency hopping waveforms to use depends on channel characteristics and signal processing complexity. In MIMO sonar applications, where the phase information of the received echoes is severely distorted due to reverberation effects of the channel, frequency hopping waveforms are a better choice. Whereas for most of the radar applications, phase coded waveforms are a better choice, due to their constant modulus property, easy generation and processing.

\section{REFERENCES}

[1] Haimovich, A.M., Blum, R.S., Cimini, L.J., "MIMO Radar with Widely Separated Antennas," IEEE Signal Processing Magazine, vol.25, Issue 1, pp.116-129, Sept. 2008
[2] Jian Li and P. Stoica, "MIMO Radar with Colocated Antennas," IEEE Signal Processing Magazine, vol.24, no.5, pp.106-114, Sept. 2007

[3] P. Woodward, Probability and Information Theory, with Applications to Radar. New York: Pergamon, 1957.

[4] G. San Antonio, D.R. Fuhrmann and F.C. Robey, "MIMO radar ambiguity functions," IEEE Journal of Selected Topics in Signal Processing, pp. 167-177, Jul. 2007.

[5] Chun-Yang Chen and P. P. Vaidyanathan, "MIMO Radar Ambiguity Properties and Optimization Using Frequency-Hopping Waveforms", IEEE Trans. on Signal Processing, pp. 5926-5936, Dec. 2008.

[6] N. Levanon and E. Mozeson, Radar Signals. New York: Wiley-IEEE Press, 2004.

[7] H. Deng, Polyphase code design for orthogonal netted radar systems, IEEE Trans. Signal Process. 52(11):31263135 (Nov. 2004).

[8] H. Deng, "Synthesis of binary sequences with good autocorrelation and cross-correlation properties by simulated annealing," IEEE Trans. Aerosp. Electron. Syst., vol. 32, pp. 98-107, Jan. 1996.

[9] Sharma G.V.K., Raja Rajeswari K, Four Phase Orthogonal Code Design for MIMO Radar Systems, IEEE National Conference on Communications, NCC2012, Feb 2012

[10] Bo Liu, Zishu He, Jiankui Zeng, Benyong Liu, Polyphase Orthogonal Code Design for MIMO Radar Systems, Proc. 2006 CIE Int. Conf. Radar, Oct. 2006

[11] S. Kirkpatrick, C. D. Gelatt, and M. P. Vecchi, "Optimization by simulated annealing," Science, vol. 220, pp. 671-680, May 1983. 